\title{
Involvement of Chromatin Remodeling Genes and the Rho GTPases RhoB and CDC42 in Ovarian Clear Cell Carcinoma
}

OPEN ACCESS

Edited by:

Margaret Jane Currie,

University of Otago, New Zealand

Reviewed by:

Nhan Le Tran

Mayo Clinic Arizona, USA

Cristin Gregor Print,

University of Auckland,

New Zealand

${ }^{*}$ Correspondence:

Ingrid A. Hedenfalk

ingrid.hedenfalk@med.lu.se

${ }^{+}$Present address:

Katarina Bartuma,

Department of Vitreoretinal

Diseases, St. Erik Eye Hospital,

Karolinska Institutet,

Stockholm, Sweden

Specialty section: This article was submitted

to Cancer Genetics,

a section of the journal

Frontiers in Oncology

Received: 16 February 2017 Accepted: 10 May 2017

Published: 29 May 2017

Citation:

Arildsen NS, Jönsson JM, Bartuma K, Ebbesson A, Westbom-

Fremer S, Måsbäck A, Malander S, Nilbert M and Hedenfalk IA (2017) Involvement of Chromatin Remodeling Genes and the Rho GTPases RhoB and CDC42 in Ovarian Clear Cell Carcinoma.

Front. Oncol. 7:109. doi: 10.3389/fonc.2017.00109

\section{Nicolai Skovbjerg Arildsen ${ }^{1}$, Jenny-Maria Jönsson ${ }^{1}$, Katarina Bartuma ${ }^{1 \dagger}$, Anna Ebbesson ${ }^{1,2}$, Sofia Westbom-Fremer ${ }^{3}$, Anna Måsbäck ${ }^{3}$, Susanne Malander ${ }^{1}$, Mef Nilbert ${ }^{1,4}$ and Ingrid A. Hedenfalk ${ }^{1,2 *}$}

'Division of Oncology and Pathology, Department of Clinical Sciences, Skåne University Hospital, Lund University, Lund, Sweden, ${ }^{2}$ CREATE Health Strategic Center for Translational Cancer Research, Lund University, Lund, Sweden, ${ }^{3}$ Department of Clinical Pathology, Division of Laboratory Medicine, Skåne University Hospital, Lund, Sweden, ${ }^{4}$ Clinical Research Centre, Hvidovre University Hospital, Copenhagen University, Hvidovre, Denmark

Objective: Ovarian clear cell carcinomas (OCCCs) constitute a rare ovarian cancer subtype with distinct clinical features, but may nonetheless be difficult to distinguish morphologically from other subtypes. There is limited knowledge of genetic events driving OCCC tumorigenesis beyond ARID1A, which is reportedly mutated in 30-50\% of OCCCs. We aimed to further characterize OCCCs by combined global transcriptional profiling and targeted deep sequencing of a panel of well-established cancer genes. Increased knowledge of OCCC-specific genetic aberrations may help in guiding development of targeted treatments and ultimately improve patient outcome.

Methods: Gene expression profiling of formalin-fixed, paraffin-embedded (FFPE) tissue from a cohort of the major ovarian cancer subtypes (cohort $1 ; n=67$ ) was performed using whole-genome cDNA-mediated Annealing, Selection, extension and Ligation (WG-DASL) bead arrays, followed by pathway, gene module score, and gene ontology analyses, respectively. A second FFPE cohort of 10 primary OCCCs was analyzed by targeted DNA sequencing of a panel of 60 cancer-related genes (cohort 2). Nonsynonymous and non-sense variants affecting single-nucleotide variations and insertions or deletions were further analyzed. A tissue microarray of 43 OCCCs (cohort 3) was used for validation by immunohistochemistry and chromogenic in situ hybridization.

Results: Gene expression analyses revealed a distinct OCCC profile compared to other histological subtypes, with, e.g., ERBB2, TFAP2A, and genes related to cytoskeletal actin regulation being overexpressed in OCCC. ERBB2 was, however, not overexpressed on the protein level and ERBB2 amplification was rare in the validation cohort. Targeted deep sequencing revealed non-synonymous variants or insertions/deletions in 11/60 cancer-related genes. Genes involved in chromatin remodeling, including ARID1A, SPOP, and KMT2D were frequently mutated across OCCC tumors.

Conclusion: OCCCs appear genetically heterogeneous, but harbor frequent alterations in chromatin remodeling genes. Overexpression of TFAP2A and ERBB2 was 
observed on the mRNA level in relation to other ovarian cancer subtypes. However, overexpression of ERBB2 was not reflected by HER2 amplification or protein overexpression in the OCCC validation cohort. In addition, Rho GTPase-dependent actin organization may also play a role in OCCC pathogenesis and warrants further investigation. The distinct biological features of OCCC discovered here may provide a basis for novel targeted treatment strategies.

Keywords: clear cell ovarian cancer, gene expression profiling, deep sequencing, inter-tumor heterogeneity, chromatin modification, Rho GTPase, ERBB2

\section{INTRODUCTION}

Ovarian clear cell carcinomas (OCCCs) represent a distinct histological and clinical subset accounting for $5-10 \%$ of epithelial ovarian cancers (EOCs), but among Asian women, the rate is at least $15 \%$. In general, EOC is characterized by an adverse prognosis, with a relative 5 -year survival of $<50 \%$ (1). The survival of EOC is influenced by stage, age at diagnosis, histological grade, residual tumor burden after surgery, and, not least, histopathological subtype (2-4). Of the four major histopathological subtypes [serous, endometrioid (EM), mucinous (MUC), and clear cell ovarian cancers], OCCCs stand out as a group with distinct clinical characteristics (5). They are considered type 1 tumors, arising gradually through progression from benign precursors associated with endometriosis, and eventually developing into invasive carcinomas $(6,7)$. Although often diagnosed in early stages, and with a younger median age at diagnosis compared to, e.g., high-grade serous ovarian carcinomas, stage III-IV OCCC confers an inferior prognosis relative to stage-matched serous tumors $(8,9)$. The outcome for patients with OCCC, regardless of stage, is also worse than for patients with EM ovarian cancer (10). The inferior prognosis may be related to a high prevalence of primary chemotherapy resistance, primarily to platinum-based drugs. This may be due to both a low proliferation rate (a prototypic type 1 tumor feature) and intrinsic resistance mechanisms such as expression of apoptosis regulators, e.g., p53 and p21, although this is not entirely clear [(11), reviewed in Ref. (12)].

Regardless of their relatively distinct clinical features, OCCCs may be difficult to distinguish histopathologically from other epithelial ovarian tumors, especially of the EM and high-grade serous subtypes. Immunohistochemical (IHC) markers such as Napsin A and HNF1 $\beta$ are useful tools; nevertheless, the diagnosis may sometimes be difficult to confirm using IHC alone $(13,14)$. Using global gene expression analysis, however, distinct transcriptional signatures of OCCC have been described, capturing not only the morphological appearance but also the biological behavior of this ovarian cancer subtype $(15,16)$. Furthermore, ARID1A, involved in chromatin remodeling, has been reported to be mutated in a subset of OCCCs, and other components of the chromatin remodeling system have also recently been reported to be affected in EM and high-grade serous ovarian cancer [(17-19), reviewed in Ref. (20)]. Table 1 summarizes the most common aberrations reported in OCCC.

In the present study, we aimed to further characterize OCCCs and their histotype-specific genetic aberrations using global gene expression profiling as well as targeted deep sequencing of a set of well-established cancer genes. Increased knowledge of genetic aberrations in OCCCs may help to guide future development of targeted treatments and clinical trials.

\section{MATERIALS AND METHODS}

\section{Tumor Material}

The tumor material used in the present study was retrieved from three different cohorts and is referred to throughout as cohorts 1,2 , and 3. Cohort 1 comprises 72 ovarian carcinomas of mixed histopathologic subtypes, partly outlined in Jönsson et al. (29). The tumors in cohort 1 were collected among Swedish and Danish Lynch syndrome carriers $(n=28)$ and Swedish sporadic cases $(n=44)$. Formalin-fixed, paraffin-embedded (FFPE) tissue was available from all tumors. Cohort 2 consists of 11 OCCCs, 9 of which were obtained from the Skåne University Hospital (Sweden) ovarian tumor biobank, and 2 of which overlapped with cohort 1. FFPE tissue was available from all tumors. Cohort 3 consists of 43 OCCCs from Skåne University Hospital arranged in a tissue microarray (TMA). Clinicopathological data for all cohorts are summarized in Table 2. All tumor samples were collected at primary surgery, and the patients had not received prior chemotherapy. Histopathological subtype and grade were determined according to Silverberg and the WHO 2003 classification, which was used at the time of diagnosis $(30,31)$. For tumors in cohorts

TABLE 1 | Common alterations in ovarian clear cell carcinoma.

\begin{tabular}{lcc}
\hline Genomic alterations & Altered in & Reference \\
\hline ARID1A (loss of function) & $\sim 50 \%$ & $(18)$ \\
PIK3CA (gain of function) & $\sim 50 \%$ & $(21,22)$ \\
TP53 (loss of function) & $\sim 25 \%$ & $(21)$ \\
KRAS (loss of function) & $\sim 10 \%$ & $(21,23)$ \\
CMET (gain of function) & $<5 \%$ & $(21)$ \\
PTEN (loss of function) & $<5 \%$ & $(21)$ \\
mRNA expression & & \\
FXYD2 (upregulated) & $\mathrm{NA}$ & $(24)$ \\
WT1 (downregulated) & $\mathrm{NA}$ & $(24)$ \\
TFAP2A (upregulated) & $\mathrm{NA}$ & $(25)$ \\
ESR1 (downregulated) & $\mathrm{NA}$ & $(25)$ \\
Protein expression & & \\
Napsin A (amplification) & $\sim 80 \%$ & $(13)$ \\
HNF-1B (amplification) & $>80 \%$ & $(26)$ \\
AKT (amplification) & $\sim 70 \%$ & $(27)$ \\
PTEN (loss) & $\sim 50 \%$ & $(21)$ \\
cMET (loss) & $\sim 25 \%$ & $(21)$ \\
HER2 (amplification) & $\sim 12 \%$ & $(28)$
\end{tabular}


TABLE 2 | Clinical characteristics of ovarian clear cell carcinomas (OCCCs) in the study cohorts.

\begin{tabular}{|c|c|c|c|c|}
\hline & \multicolumn{2}{|c|}{ Cohort 1} & \multirow{2}{*}{$\frac{\text { Cohort } 2}{\text { occC }}$} & \multirow{2}{*}{$\begin{array}{c}\text { Cohort } 3 \\
\text { OccC }\end{array}$} \\
\hline & All tumors & occc & & \\
\hline & $n=67$ & $n=15$ & $n=10$ & $n=43$ \\
\hline \multicolumn{5}{|c|}{ Age at diagnosis (years) } \\
\hline Median & 51 & 48 & 66.5 & 63 \\
\hline Range & $27-78$ & $34-60$ & $51-90$ & $41-90$ \\
\hline \multicolumn{5}{|l|}{ Stage, $n(\%)$} \\
\hline । & $28(46)$ & $13(92)$ & $7(78)$ & $27(63)$ \\
\hline ॥ & $9(15)$ & 0 & $1(11)$ & $6(14)$ \\
\hline III & $20(33)$ & $1(8)$ & $1(11)$ & $9(21)$ \\
\hline IV & $4(7)$ & 0 & 0 & $1(2)$ \\
\hline Missing & 6 & 1 & 1 & 0 \\
\hline \multicolumn{5}{|c|}{ Age of formalin-fixed, paraffin-embedded blocks (years) } \\
\hline Median & 12 & 19 & 4.5 & 8 \\
\hline Range & $3-54$ & $3-38$ & $2-16$ & $8-13$ \\
\hline \multicolumn{5}{|c|}{ Histology, $n$ (\%) } \\
\hline Clear cell & $15(22)$ & $15(100)$ & $10(100)$ & $43(100)$ \\
\hline Serous & $31(46)$ & - & - & - \\
\hline Endometrioid & $18(27)$ & - & - & - \\
\hline Mucinous & $3(4)$ & - & - & - \\
\hline
\end{tabular}

2 and 3, the histopathological subtype was determined according to the more recent WHO 2014 classification (32). All tumors were staged according to the International Federation of Gynecology and Obstetrics criteria (33). Ethical approval for the study was granted from the ethics committee in Region Hovedstaden, Denmark, and from the Lund University ethics committee, Sweden, and all patients provided written, informed consent.

\section{RNA Extraction and Gene Expression Analysis}

RNA was extracted from all FFPE samples in cohort $1(n=72$ ovarian carcinomas). As outlined previously, non-necrotic tumor areas with $>70 \%$ tumor cell content were selected from three to five $10 \mu \mathrm{m}$ sections of Hematoxylin and Eosin stained tissue per tumor and RNA was extracted from these areas using the High Pure RNA Paraffin Kit (Roche, Castle Hill, Australia) (29). RNA concentrations were determined using a NanoDrop Spectrophotometer (NanoDrop Technologies, Wilmington, DE, USA); 68/72 tumors met the quality criteria (300 ng of RNA with a 260/280 ratio $>1.8$ ). Gene expression analyses were performed at the SCIBLU Genomics Centre, Lund University, Sweden. The cDNA-mediated Annealing, Selection, extension and Ligation (WG-DASL) assay (Illumina Inc., San Diego, CA, USA), containing 24,526 probes representing 18,626 unique genes, was used for whole-genome expression analysis. The samples were randomized on the chips and were profiled following the manufacturer's instructions. BeadChips were then scanned on a BeadArray ${ }^{\mathrm{TM}}$ Reader using BeadScan software (v4.2), during which fluorescence intensities were read and images extracted. A raw average signal intensity $>250$ for each probe and $>8,000$ detected genes/sample were required for further analysis; 67/68 samples met these criteria. The raw data are freely available in NCBI's Gene Expression Omnibus (34) through GEO series accession number GSE37394. The raw data were quantile normalized, log2 transformed and subjected to a presence filter of $80 \%$ across probes, with a detection $P$-value $\leq 0.01$, leaving 12,747 probes. Biological duplicates were averaged and when multiple probes identified the same gene, the probe with the highest variance across tumors was chosen to represent the gene, resulting in 10,000 remaining probes. The data were then mean centered across samples using $\mathrm{R}$ version 3.2.2 (35). Thereafter, the data were imported into the $\mathrm{MeV}$ version 4.9.0 software (36) and a multiclass significance analysis of microarrays (SAM) analysis (37) using the histological subtypes as groups (serous, EM, MUC, and clear cell) was performed. A SAM analysis, using 1,000 random permutations, was used to identify the $5 \%$ most significant differentially expressed genes between subgroups, with a false discovery rate $(\mathrm{FDR})<5 \%$. OCCCs were compared to the other subtypes individually using the same settings. Pearson correlation was used for distance metric selection and complete linkage was used for cluster generation. Enrichments of gene ontology (GO) terms were explored using the online Panther gene list analysis tool with default settings for Homo sapiens, using a binomial statistical overrepresentation test and Bonferroni correction for multiple testing with the 10,000 genes as background (38). GO terms were compared using the online Gene Semantic Similarity Analysis and Measurement tool (G-SESAME) with default settings (39), generating a semantic similarity score between GO terms of 0 (low similarity) to 1 (high similarity). Gene set analysis using gene sets from the REACTOME database [1,764 pathways, ConsensusPathD (40)] was performed using the GS-Reg package version 1.8 in $\mathrm{R}$ with 1,000 permutations, a minimum gene count of 7 and a $P$-value cutoff of 0.001 for all genes (41). Genes from the SAM analysis were analyzed using the ConsensusPathDB, with a minimum gene count of 7 and a $q$-value cutoff of 0.05 for REACTOME pathways. Venn diagrams were generated using the online Venn Diagram tool from the Bioinformatics and Evolutionary Genomics web page. ${ }^{1}$ Gene module score comparisons according to gene modules defined by Desmedt et al. were performed as previously described $(42,43)$. Statistical tests (Kruskal-Wallace and Dunn's test) and boxplots were performed using $\mathrm{R}$ version 3.2.2.

\section{DNA Extraction and Targeted Sequencing}

DNA was extracted from all FFPE samples in cohort $2(n=11$ OCCCs) using the AllPrep DNA/RNA FFPE Kit (Qiagen, Venlo, Netherlands). Two $10 \mu \mathrm{m}$ tissue sections/tumor were used for DNA extraction and $>1 \mu \mathrm{g}$ DNA was obtained from all samples. Three micrometer hematoxylin stained sections, retrieved immediately before the sections used for DNA extraction, were reviewed by a gynecological pathologist (SWF) to establish tumor cellularity, which ranged from 65 to $99 \%$, with a median of $90 \%$ (data not shown). DNA concentrations were measured using the Qubit Fluorometric Quantitation ${ }^{\circledR}$ (Life Technologies, Thermo Fisher Scientific, Waltham, MA, USA), and a qPCR-based DNA quality control was performed using the Trusight Tumor Sample Preparation Kit (Illumina Inc.), with $\Delta$ CT values $<6.5$ considered

${ }^{1}$ http://bioinformatics.psb.ugent.be/webtools/Venn/. 
sufficient. One sample was excluded due to poor DNA quality. For the remaining 10 samples, $600 \mathrm{ng}$ genomic DNA/sample $(50 \mathrm{ng} / \mu \mathrm{l})$ was used for targeted DNA sequencing. Sample preparation and sequencing was performed at Oxford Gene Technology ${ }^{\mathrm{TM}}$ (OGT, Oxford, Great Britain). The Next-Generation Sequencing SureSeq ${ }^{\mathrm{TM}}$ solid tumor panel, a hybridization-based enrichment tool targeting all codons of the exons in the included genes, was used. The gene panel consists of 60 key cancer genes and has been validated for research use on FFPE samples ${ }^{2}$ (Table S1 in Supplementary Material).

Alignment was performed using the human build GRCh37 reference genome and Burrows-Wheeler Aligner (version 0.7.10). Local realignment was carried out using the Genome Analysis Tool Kit (GATK, version 1.6), and duplicate reads were removed using Picard version 1.107. Variant calling was performed using the BCbio-nextgen tumor only prioritization pipeline ${ }^{3}$ with Mutect2 from GATK as variant caller and default settings (44). Variants were annotated using the Variant Effect Predictor (45). Variants were classified using the GEnome MINIng (GEMINI) framework to filter out possible germline variants (46), with a variant allele frequency filter cutoff of $>5 \%$.

\section{IHC and Chromogenic In Situ Hybridization (CISH)}

Immunohistochemical and CISH evaluation of ERBB2 were performed using standard procedures for HER2 testing in breast cancer. The anti-HER2/neu rabbit monoclonal antibody 4B5 (Ventana, Tucson, AZ, USA) was used for IHC and the 2,4-dinitrophenyl (DNP)-labeled HER2 probe and digoxigenin (DIG)-labeled Chr17 were used for CISH. The HER2 gene and Chr17 signals were detected using the ultraView SISH DNP and ultraView Red ISH DIG detection kits (Ventana). Tumors with an IHC score of $3+$ and/or a gene/centromere ratio $\geq 2$ were considered positive (Figure S1 in Supplementary Material). AntiAKT-pS473 (Dako, Denmark) staining was performed using Dako EnVision $^{\mathrm{TM}}$ System/HRP. A 1:10 dilution was used with Dako Retrieval Solution ( $\mathrm{pH}$ 9). Any staining was considered positive.

\section{RESULTS}

\section{Gene Expression Analyses Reveal Distinct OCCC Profiles and Involvement of Rho GTPases}

We wanted to investigate the gene expression profiles of OCCC compared to the other ovarian cancer subtypes. In a previous study of Lynch syndrome-associated ovarian cancer, we reported a distinct gene expression pattern of OCCC compared to other subtypes using unsupervised hierarchical clustering. This was found to be irrespective of Lynch status (29). Hence, we performed a supervised multiclass SAM analysis without a variance filter using all 10,000 genes and histological subtypes as groups, thereby identifying 505 differentially expressed

\footnotetext{
${ }^{2}$ http://www.ogt.co.uk/products/915_sureseq_solid_tumour_panel. ${ }^{3}$ https://github.com/chapmanb/bcbio-nextgen.
}

genes (FDR $<5 \%)$. OCCCs were found to display a distinct transcriptional pattern compared to all the other histological subtypes (Figure 1A). The pathway analysis revealed four altered pathways (Table 3), with the extracellular matrix (ECM) organization, axon guidance, and developmental biology pathways exhibiting many genes in common. We next applied a variance filter to select the 2,500 genes with the highest variance across tumors. This was done to select as many targets as possible throughout the signaling network hierarchy, as proposed by Komurov and Ram (47). This SAM analysis identified 504 significant genes (FDR $<10 \%$ ), and the subsequent pathway enrichment analysis revealed genes belonging to both the NCAM signaling and TFAP2A transcription pathways, as well as pathways involved in MAPK signaling. In addition, pathways connected to ECM organization, axon guidance, and developmental biology were also differentially expressed (Table 3). Although three pathways were found in common between the two SAM analyses, only $48 \%(271 / 504)$ of the genes overlapped (data not shown).

To further explore this finding, we performed a gene set analysis of the whole dataset (10,000 genes), revealing genes of the axon guidance pathway to be differentially expressed between OCCC and the other subtypes combined $(P<0.001,156 / 251$ genes in pathway present in dataset). However, when OCCCs were compared to the other subtypes individually, the genes of the axon guidance pathway were only found to be differentially expressed between the OCCC and the EM subtypes $(P<0.001$; File S1 in Supplementary Material).

Comparing the individual differentially expressed pathways between OCCC and the other subtypes, we found the highest number of differentially expressed pathways when comparing OCCC to the EM subtype. A total of 324 pathways, encompassing 4,599 genes, included genes that were differentially expressed between the OCCC and EM subtypes (Figure 2). However, a significant overlap of genes between 295 of these pathways (91\%) was observed. Furthermore, several pathways were found to be general and were probably a result of these pathways containing other smaller pathways, resulting in an elevated number of significant pathways. However, 30\% (96/324) of these pathways had genes which were differentially expressed between OCCC and the serous and EM subtypes combined. Among these 96 pathways were the "TFAP2 (AP-2) family regulates transcription of growth factors and their receptors" pathway, the "Chromatin organization" pathway, and several PI3-kinase and AKT associated pathways (File S1 and File S2 in Supplementary Material). A comparison of the genes in the differentially expressed pathways revealed three genes of interest: the Rho GTPase CDC42, the growth factor receptor $E R B B 2$, and the transcription factor TFAP2A. These genes were differentially expressed between subtypes, with overexpression in OCCC (Figure 1B), and were also present in many of the altered pathways in both the SAM and whole gene set analyses. Genes in the Rho GTPase pathways were also differentially expressed between OCCC and both the EM and serous subtypes; however, no direct overlap in pathways between subtypes was observed.

The overexpression of ERBB2 in the OCCC subtype prompted us to investigate the protein expression and copy number status 

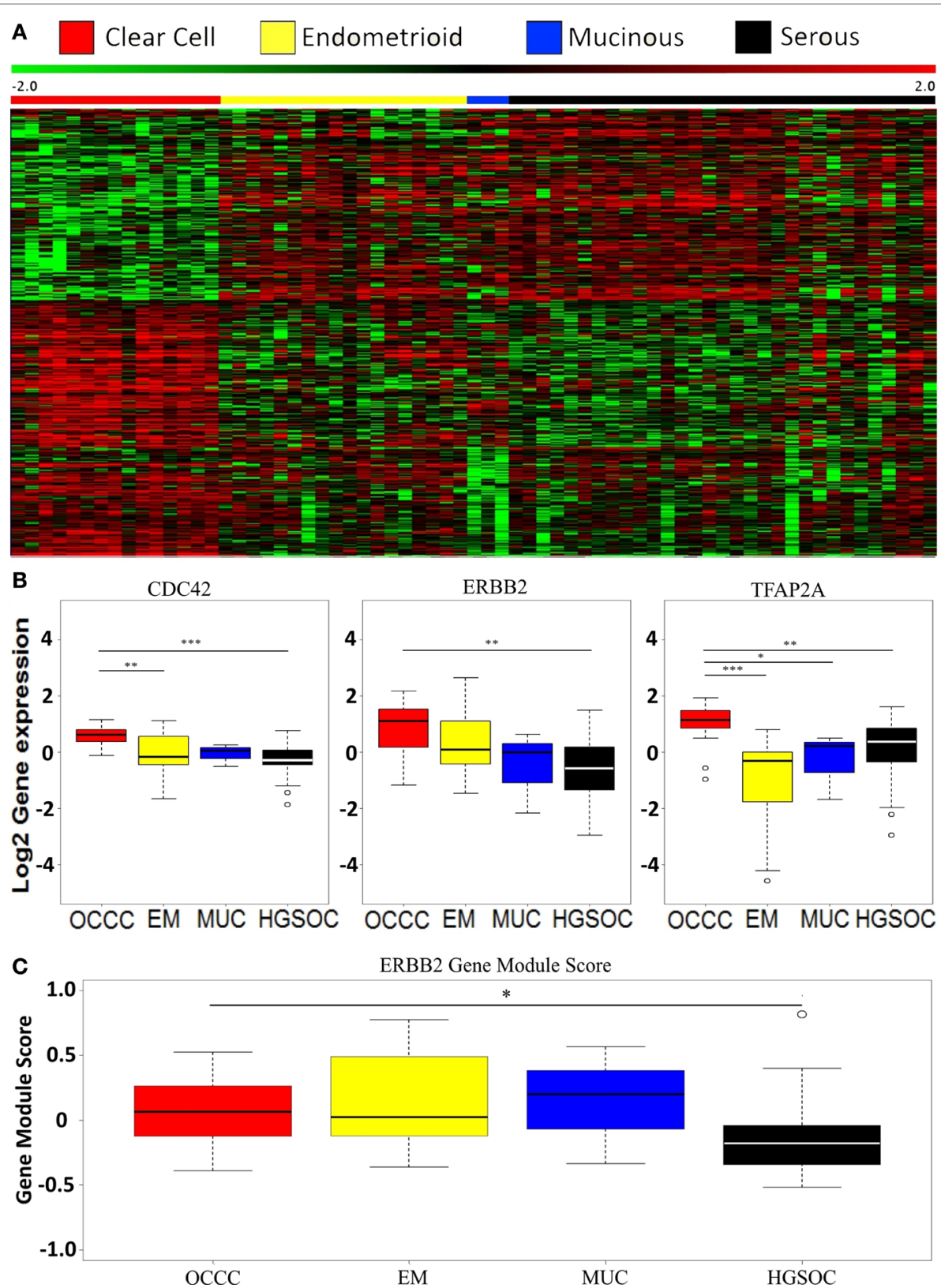

FIGURE 1 | Global gene expression profiles in ovarian cancer. (A) Heatmap of the 505 most significant differentially expressed genes based on a supervised multiclass significance analysis of microarrays analysis comparing the four histopathological subtypes (serous, EM, MUC, and clear cell) in cohort 1. (B) Boxplots illustrating differential expression of $C D C 42$, ERBB2, and TFAP2A between the histological subtypes using Dunn's test for difference. ${ }^{\star} P<0.05$; ${ }^{\star \star} P<0.01$; ${ }^{\star \star \star} P<0.001$. (C) Boxplot illustrating ERBB2 gene module scores in the histological subtypes as defined by Desmedt et al. using Dunn's test for difference. ${ }^{*} P<0.05$. OCCC, ovarian clear cell carcinoma; EM, endometrioid; MUC: mucinous; HGSOC: high-grade serous ovarian cancer.

of ERBB2 using a TMA. Only one of 43 OCCC tumors (2\%) showed overexpression and amplification of ERBB2 (Figure S1 in Supplementary Material), and one tumor displayed amplification (ratio $\geq 2$ ) with weak protein expression $(1+$ ). Three tumors (7\%) displayed weak protein expression $(1+)$ without amplification. This suggests that while ERBB2 may be overexpressed in OCCC on the mRNA level relative to other histological subtypes of ovarian cancer, this does not result in overexpression on the protein level, nor does it appear to be caused by amplification of the ERBB2 gene. Further support of this was found when applying a module score for the ERBB2 pathway from breast cancer to our data $(P=0.2896$, two sided $t$-test, 17/28 genes present) (43), which did not provide evidence for ERBB2 mediated activity in OCCC (Figure 1C). However, there was a significant difference between the OCCC and serous subtypes ( $P=0.0147$, Dunn's test, $17 / 28$ genes present), with a lower module score in the serous subtype, in line with a previous study from our group (42). 
TABLE 3 | Pathway enrichment of differentially expressed genes identified using significance analysis of microarrays analysis with/ without a variance filter.

\begin{tabular}{|c|c|}
\hline Pathway ${ }^{a}$ & $q$-Value \\
\hline \multicolumn{2}{|l|}{ No variance filter } \\
\hline Extracellular matrix (ECM) organization & 0.026 \\
\hline Axon guidance & 0.045 \\
\hline Developmental biology & 0.045 \\
\hline $\begin{array}{l}\text { Transport of glucose and other sugars, bile salts and organic } \\
\text { acids, metal ions and amine compounds }\end{array}$ & 0.045 \\
\hline \multicolumn{2}{|l|}{ Variance filter } \\
\hline NCAM signaling for neurite out-growth & 0.012 \\
\hline NCAM1 interactions & 0.015 \\
\hline $\begin{array}{l}\text { Transcriptional regulation by the AP-2 (TFAP2) family of } \\
\text { transcription factors }\end{array}$ & 0.017 \\
\hline ECM organization & 0.017 \\
\hline ECM proteoglycans & 0.024 \\
\hline GPCR ligand binding & 0.039 \\
\hline Axon guidance & 0.039 \\
\hline Signaling by GPCR & 0.039 \\
\hline RAF/MAP kinase cascade & 0.039 \\
\hline SHC1 events in EGFR signaling & 0.039 \\
\hline SOS-mediated signaling & 0.039 \\
\hline GRB2 events in EGFR signaling & 0.039 \\
\hline Gastrin-CREB signaling pathway via PKC and MAPK & 0.039 \\
\hline Signaling to p38 via RIT and RIN & 0.039 \\
\hline ARMS-mediated activation & 0.039 \\
\hline MAPK family signaling cascades & 0.039 \\
\hline Frs2-mediated activation & 0.039 \\
\hline MAPK1/MAPK3 signaling & 0.039 \\
\hline Prolonged ERK activation events & 0.043 \\
\hline Signaling by Leptin & 0.045 \\
\hline Signaling to RAS & 0.045 \\
\hline Developmental biology & 0.045 \\
\hline Interleukin receptor SHC signaling & 0.047 \\
\hline VEGFR2-mediated cell proliferation & 0.049 \\
\hline
\end{tabular}

aMinimum gene presence: 7; false discovery rate $<0.05$.

In a previous study, we evaluated the protein expression of mTOR, PTEN, and EGFR in OCCCs in cohort $1(n=12)$. EGFR was expressed in two tumors (17\%), PTEN in four tumors $(33 \%)$, and mTOR in 7 tumors (58\%) (29). pAKT was evaluated using the previously mentioned TMA of 43 OCCCs. pAKT protein expression was observed in 23 tumors (58\%).

Next, we performed a GO term similarity score analysis using the genes from the SAM analysis without variance filter and compared those to a previous study of OCCC (including the clear cell, serous and EM subtypes) (16) using G-SESAME. The similarity score was 0.683 , confirming our findings, and GO terms above this threshold were related to anatomical structure development, cell differentiation, and signaling (Table S2 in Supplementary Material).

Taken together, these findings indicate that different mechanisms involved in structural modifications, such as Rho GTPases and chromatin organization genes, may play an important role in tumor development and progression in OCCC. In addition, upregulation of the transcription factor TFAP2A may be involved in the distinct transcriptional differences observed between OCCC and other subtypes. Furthermore, differential activation of the PI3K and mTOR pathways, implicated in the development of type I ovarian carcinomas, between OCCC and both EM and

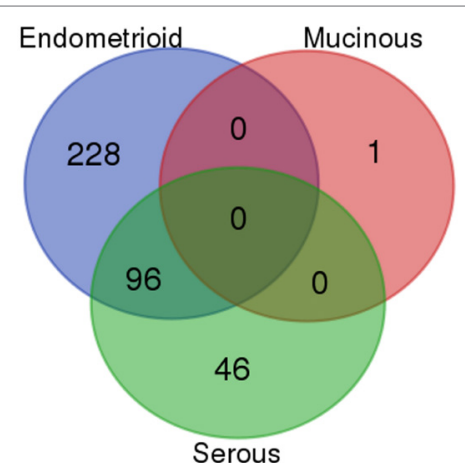

FIGURE 2 | Venn diagram of the overlap of differentially expressed Reactome pathways between ovarian cancer subtypes. The number of overlapping Reactome pathways from the gene set analysis of ovarian clear cell carcinoma compared to the high-grade serous, mucinous, and endometrioid subtypes, respectively.

serous tumors, may indicate deregulation of the PI3K/mTOR/ AKT pathway in OCCC.

\section{Targeted Deep Sequencing Uncovers Variants in Genes Involved in Chromatin Remodeling and Transcriptional Regulation in OCCC}

The transcriptional differences observed across the ovarian cancer subtypes, specifically the appearance of a distinct OCCC gene expression profile, prompted further analyses of the potential underlying mutational drivers of OCCC. We therefore performed targeted deep sequencing of 60 cancer-related genes in the 10 OCCCs from cohort 2 ; $>99 \%$ of the target bases had at least $30 \mathrm{X}$ coverage across all 10 samples, with a mean target coverage of 534X (range 314X-698X). A total of 8,595 variants were called, spanning 3,849 unique variants across the 10 tumors. Of these, 1,244 were tagged by GEMINI as potential germline variants, while Mutect2 filtered out 7,319 variants, leaving 32 variants $(0.3 \%)$ using a minor allele frequency cutoff of $5 \%$.

The 32 mutations were manually compared to the COSMIC, ExAC, and ENSEMBL (dbSNP148) databases to identify previously reported variants and filter out variants with a global minor allele frequency $>0.0001$ (i.e., $1 / 10,000$, suspected to be germline SNPs). Twenty probable somatic variants were identified in 11 genes, with a variant range from 0 to 5 (median 2), supporting the notion of a genomically stable subtype. Two variants were inframe indels, 10 were missense variants and eight were truncating variants. Forty-five percent (9/20) of the variants have not been previously reported (Figure $\mathbf{3 A}$ and Table 4 ). Using the gene family nomenclature provided by the online Molecular Signatures Database (MSigDB V5.1; Broad Institute, MA), 2 of these genes are tumor suppressors (CDKN2A, TP53), 6 are oncogenes (ERBB2, PIK3CA, KRAS, ALK, NOTCH1, ROS1), 2 are transcription factors (TP53, SPOP), and 3 are protein kinases (ERBB2, ALK, ROS1). Next, the PantherDB online tool was used to explore potential affected pathways. Several pathways, 


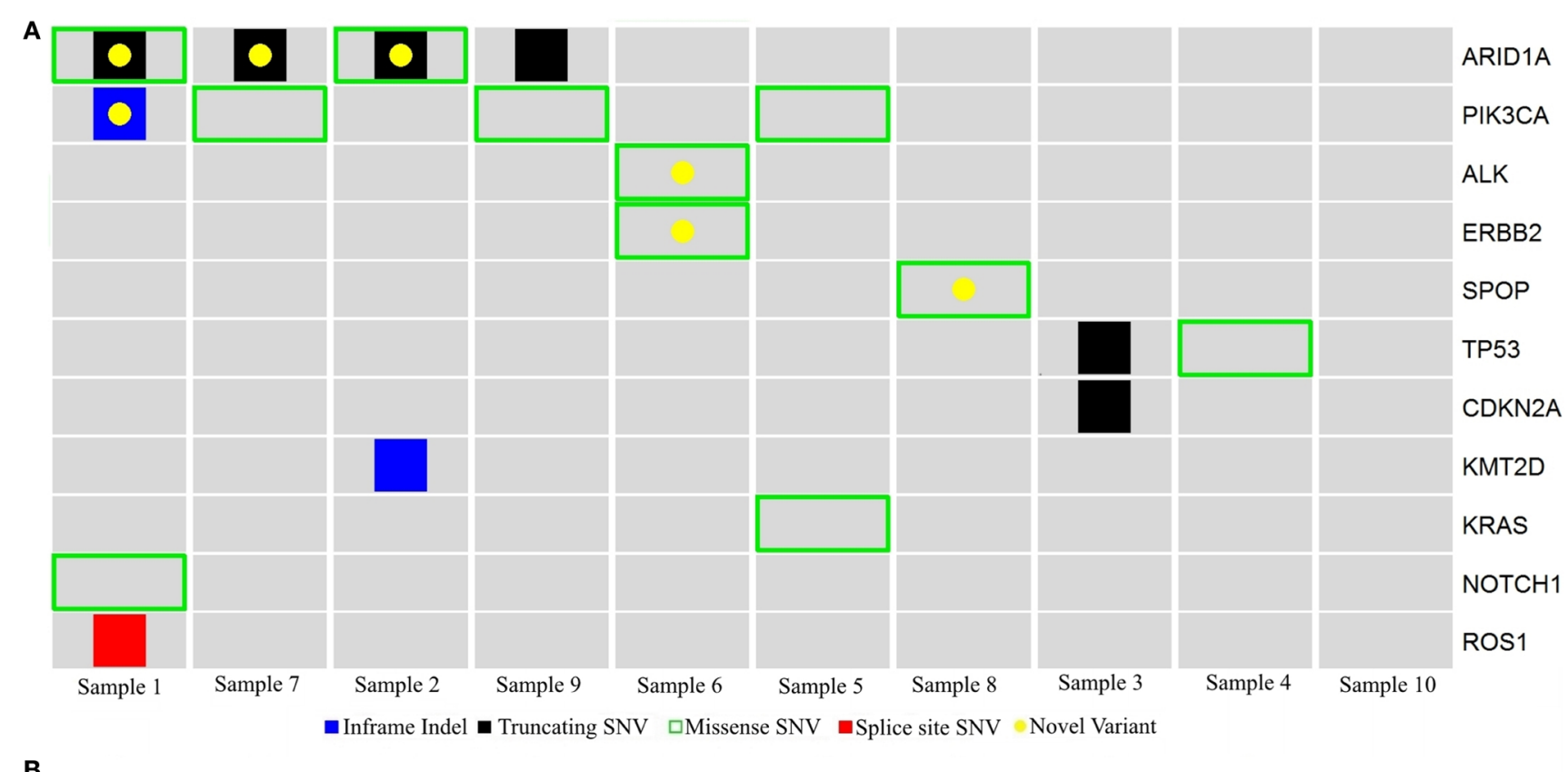

B

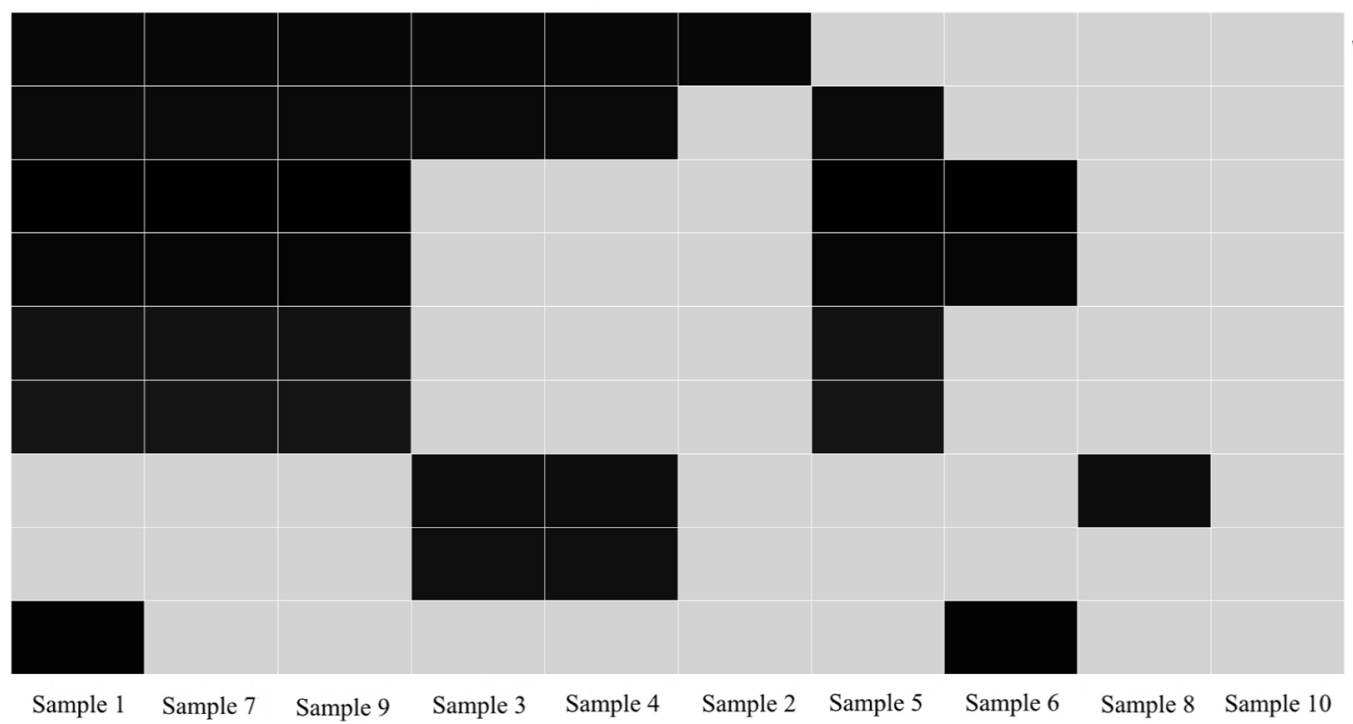

WNT signaling

P53 associated signaling

Oncogene

EGF receptor signaling

PIK3CA/KRAS signaling

Angiogenesis

Transcription factors

Tumor suppressor

Protein kinases

FIGURE 3 | Schematic diagram of gene variants in ovarian clear cell carcinoma. (A) Illustration of probable somatic variants in genes (rows) across tumors (columns) in cohort 2. Red, splice site SNV; black, truncating SNV; green, missense SNV; blue, inframe indels; yellow, unreported variants. (B) Summary of pathways and gene families (rows) affected by probable somatic variants across tumors (columns) in cohort 2.

including the TP53, WNT, EGF, and PIK3CA/KRAS pathways were found to be affected (Figure 3B).

$A R I D 1 A$ and PIK3CA variants, previously identified in OCCC, were present in 4/10 (40\%) tumors, and co-occurred in three tumors (30\%). Samples 2 and 9 had $>1$ ARID1A variant each. The six ARID1A variants included three small indels and three missense variants; five of these variants resulted in protein truncation downstream of the variation site (Table 4). Only one variant, the truncating variant Gln2176 in Sample 9, has previously been reported, although not in ovarian cancer. Of the three PIK3CA variants, the missense variant His1047Arg occurred in two samples and has been associated with ovarian cancer in 91 tumors in the COSMIC database. Two variants had previously been reported in COSMIC, while the EDLLNPI453del variant in Sample 1 has not been reported.

The ERBB2 variant (p.989I > M) has not previously been reported in any of the databases used in this study. However, it is located outside the functional domains of the ERBB2 gene, and the significance of this finding is therefore unknown (data not shown).

Two TP53 single nucleotide deletion variants were found, both of which are reported in COSMIC (Table 4). The missense variant 
TABLE 4 | Somatic variants in ovarian clear cell carcinoma and their reported RsID and status in the COSMIC database.

\begin{tabular}{|c|c|c|c|c|}
\hline & Gene & Alteration & RsID & cosmic \\
\hline Sample 1 & ARID1A & Gln1614* & NR & NR \\
\hline Sample 1 & NOTCH1 & N280S & rs367825691 & NR \\
\hline Sample 1 & PIK3CA & EDLLNPI453del & NR & NR \\
\hline Sample 1 & ROS1 & V1881_splice & rs772648589 & NR \\
\hline Sample 2 & ARID1A & $\mathrm{P} 476^{\star}$ & NR & NR \\
\hline Sample 2 & ARID1A & Leu1054Phe & NR & NR \\
\hline Sample 2 & KMT2D & Gln3745dup & rs775492040 & NR \\
\hline Sample 3 & CDKN2A ${ }^{a}$ & Glu69* & rs121913383 & CoSM13281 \\
\hline Sample 3 & TP53 ${ }^{a}$ & Asn268* & NR & cosM6583 \\
\hline Sample 4 & TP53 & Arg181Cys & rs587782596 & CoSM11090 \\
\hline Sample 5 & KRAS & Gly12Val & rs121913529 & COSM520 \\
\hline Sample 5 & PIK3CA & His1047Arg & rs121913279 & COSM775 \\
\hline Sample 6 & ALK & Gly926Arg & NR & NR \\
\hline Sample 6 & ERBB2 & Ile989Met & NR & NR \\
\hline Sample 7 & ARID1A ${ }^{a}$ & Tyr1055* & NR & NR \\
\hline Sample 7 & PIK3CA ${ }^{a}$ & Lys111Glu & NR & CoSM13570 \\
\hline Sample 8 & SPOP & Lys115Glu & NR & NR \\
\hline Sample 9 & ARID1A & Gln537* & NR & NR \\
\hline Sample 9 & ARID1A & Gln2176* & NR & COSM1582021 \\
\hline Sample 9 & PIK3CA & His1047Arg & rs121913279 & cosm775 \\
\hline
\end{tabular}

$N R$, not reported; *, truncating variant; splice, splice site variant; del, deletion. a Validated using available exome sequencing data.

Arg181Cys has been identified in breast and endometrial cancer according to COSMIC, but not in ovarian cancer.

The KRAS variant found in one tumor (c.35G > T) has previously been reported as a somatic variant in ovarian cancer (COSM520).

The CDKN2A variant has previously been identified in COSMIC, however not in ovarian cancer. Finally, variants in the genes ROS1, NOTCH1, ALK, KMT2D, and SPOP have to our knowledge not previously been reported in ovarian cancer.

In summary, genes previously reported to be mutated in OCCC, including ARID1A, PIK3CA, and ERBB2, were identified in the present cohort. Variants in samples 3 and 7 were validated using available exome data with paired tumor and blood, supporting the use of FFPE tissue for confident variant calling using strict criteria as implemented in the BCbio pipeline (Table 4). Overall, genes affected by potential somatic variants in OCCCs in the present study included KMT2D, KRAS, ARID1A, PIK3CA, TP53, ERBB2, NOTCH1, and SPOP. These genes, spanning variants over $8 / 10$ tumors, are associated with a comprehensive list of functions that relate to multiple cancer driving cellular processes. However, in common are chromatin remodeling and transcriptional regulation relying on chromatin, providing a possible explanation to a common underlying cause in OCCC pathogenesis.

\section{DISCUSSION}

Differences in the clinical behavior between the histopathological subtypes of ovarian cancer are well known, and differences have also been reported on the molecular level (16, 29-31), with OCCCs displaying remarkably distinct features including resistance to chemotherapy and distinct gene expression profiles compared to other ovarian cancer subtypes (9-11). In the present study, we investigated transcriptional and mutational landscapes in order to shed light on the underlying molecular features of these cancers.

Gene expression profiling clearly distinguished OCCCs as a unique subtype compared to other subtypes of ovarian cancer. A GO analysis comparing the genes in the present study and the OCCC study by Zorn et al. (16) revealed a similarity of GO terms linked to regulation of various biological processes often found to be deregulated in cancer (48). Interestingly, the enrichment test revealed overrepresentation of the "ECM organization," "axon guidance," and "developmental biology" pathways in the four-way SAM analysis comparing all four histological subtypes in our study. A common denominator for these pathways is the Rho family of GTPases. Rho GTPases are small GTP binding proteins highly conserved through species. They regulate a broad variety of cellular functions, including actin polymerization/organization and cell migration, and are frequently aberrantly expressed in cancer (49). CDC42 and $R h o B$, both members of this family, were significantly differentially expressed in the present study. Interestingly, a study by Canet et al. suggested that Rho GTPases may contribute to the distinct morphology of OCCC. They found lower CDC42 mRNA in OCCCs compared to the high-grade serous subtype (50), although our observations indicated the converse-higher CDC42 levels in OCCC than in the EM and high-grade serous subtypes. The discrepancy may be due to differences in stage between the two studies, with the majority of tumors in the present study being stage I, whereas the tumors in the study by Canet et al. were primarily stage III and IV $(8,9)$. However, our findings correlate well with $C D C 42$ and $R h o B$ expression levels found in the Ovarian cancer database of Cancer Science Institute Singapore (CSIOVDB), reporting significantly higher $C D C 42$ and $R h o B$ levels in OCCC compared to the other subtypes (51).

Through gene expression analysis, we observed differences in expression of genes belonging to the PI3K/mTOR/AKT pathway and this was supported by IHC stainings, which showed loss of PTEN and expression of both mTOR and pAKT in OCCC tumors. This is in line with a recent study of 521 OCCCs, which reported that the PI3K/mTOR/AKT pathway was altered in $>50 \%$ of the tumors and suggested significant cross talk with other pathways, such as the RAS/RAF/MEK/ ERK pathway (21), which was found to be differentially expressed in our study (Table 3). In support of this, protein phosphatase 2A (PP2A) has recently been suggested as a potential target for the cross talk between these two pathways (52). Taken together, with PP2A acting upstream of the actin regulating RhoA/ROCK/MLC pathway (53), these findings suggest a mechanism whereby Rho GTPases connect these pathways, possibly thereby contributing to the pathogenesis of OCCC. Using a gene set-based integrative approach, Chang et al. recently reported deregulation of pathways related to the PI3K cascade in OCCC compared to normal control tissue. They also described deregulation of the GO term "Rho guanyl nucleotide exchange factor activity," also in line with our findings (54). The gene set analysis performed here confirmed these results, as pathways related to $\mathrm{PI} 3 \mathrm{~K} / \mathrm{mTOR} / \mathrm{AKT}$ signaling, implicated 
in the development of type I carcinomas including OCCC, were also deregulated between OCCC and both the EM and serous subtypes in our data set.

$E R B B 2$ has been proposed to constitute a viable therapeutic target in OCCC based on amplification of ERBB2 with concomitant protein overexpression in 14\% of OCCC tumors in one study (55). However, although ERBB2 was upregulated compared to other subtypes in our cohort, downstream effectors of the pathway appeared unaffected, and overexpression was not observed on the protein level, suggesting that the signaling pathway may not be activated in OCCC. These findings are supported by others who have investigated HER2 expression in ovarian cancer and found no prognostic value $(56,57)$. These studies are however limited due to smallnumbers. The lack of concordance and prognostic value may however be due to the apparent inactivity of the HER2 pathway reported in the present study. The cause of this remains unclear, but incomplete amplification of the HER2 region on chromosome 17 has been reported in a subset of HER2amplified breast cancers in the absence of protein overexpression (58). In summary, our findings confirm that the $\mathrm{PI} 3 \mathrm{~K} / \mathrm{mTOR} /$ AKT pathway may play an important role in OCCC pathogenesis; however, our data also implicate Rho GTPases in this context, possibly as a mediator between several pathways involved in the development of OCCC.

Transcription factor AP2- $\alpha$ (TFAP2A) is a tumor suppressor gene acting through TP53 in breast cancer, and its expression is known to inhibit cell division (59). Higher mRNA levels of TFAP2A were found in OCCC compared to the three other subtypes, which is supported by previous findings (25). Furthermore, increased TFAP2A mRNA levels have been reported as a positive prognostic marker for overall survival in EOCs of all subtypes (60). Of interest, ERBB2 expression and pathway activation has been linked to TFAP2A expression, and the "Transcriptional regulation by the AP-2 (TFAP2) family of transcription factors" pathway, which includes $E R B B 2$, was found to be differentially expressed in our study. However, the underlying mechanism has yet to be determined.

To extend our findings, we applied targeted next-generation sequencing using the SureSeq ${ }^{\mathrm{TM}}$ solid tumor panel to a cohort of 10 OCCCs. The variant pattern revealed a heterogeneous mutational landscape, but is largely in line with what has previously been reported. The oncogene KRAS is often mutated in low proliferating, type 1 ovarian tumors (23). A single KRAS variant was found in our cohort, in line with the previously reported overall variant frequency of $7-14 \%$ in $\operatorname{OCCC}(21,23)$. Altered chromatin remodeling has been reported as a driver of tumorigenesis in OCCC, with ARID1A being involved in early stages in conjunction with PIK3CA (61). Variants in ARID1A and PIK3CA were collectively observed in five tumors, consistent with previous reports stating variant frequencies of $30-50 \%$ (18). Interestingly, in a mutant mouse model of ARIDIA dependent OCCC, concurrent activation of PIK3CA was required for tumor formation, suggesting that the SWI/SNF chromatin remodeling and PI3K pathways converge in the development of OCCC (19). Other PI3K pathway aberrations, such as loss of PTEN, may contribute to OCCC pathogenesis in the absence of PIK3CA activation.
Intriguingly, two other genes involved in chromatin remodeling, KMT2D and SPOP, were identified in the present study, suggesting that although ARID1A has been the focus of attention, other defects in the chromatin remodeling system may contribute to OCCC tumorigenesis. Importantly, mutations in the three genes were mutually exclusive. SPOP indirectly affects heterochromatin maintenance through ubiquitination of the DAXX protein, thereby contributing to, e.g., renal clear cell tumorigenesis (62). KMT2D, on the other hand, is part of the COMPASS-like complex (COMplex of Proteins ASsociated with Set1) responsible for mono-methylation of H3K4, and is recruited by, e.g., nuclear binding receptors such as the estrogen receptor and by other transcription factors such as p53, which may be significant for OCCC tumorigenesis (63).

Of the 20 potential somatic variants identified across tumors in the current study, 8 were previously reported as being somatic, while the remaining variants were considered to be potential somatic variants. The potential tumorigenic effect of these 20 variants is unclear and requires further investigation.

In conclusion, overexpression of both ERBB2 and TFAP2A is frequent in OCCC, but in the case of $E R B B 2$, does not translate to protein overexpression. The role of TFAP2A in cancer progression appears to vary according to cancer type and remains to be determined in OCCC development. Deregulation of Rho GTPases was common among OCCCs compared to the other subtypes of ovarian cancer. Rho GTPases have been the focus of attention for their role in tumorigenesis, especially in invasiveness and metastasis development. Several inhibitors targeting effectors and activators of the Rho GTPases are available, and their potential role in OCCC remains to be explored. Taken together, the complex networks in which Rho GTPases participate seem to play a critical role in OCCC pathogenesis, potentially in conjunction with the PI3K/mTOR/AKT pathway. On both the genomic and gene expression levels, chromatin remodeling and subsequent deregulation of gene expression may also play a critical role in the development and progression of OCCC. Variants in KMT2D, $A R I D 1 A$, and SPOP reflect the diversity of genomic variations in OCCC. These genes play a critical role in regulating gene expression and silencing, and the variation pattern detected may contribute to the observed resistance to chemotherapy. Further investigations are needed to elucidate the possible interplay between Rho GTPases and the PI3K/mTOR/AKT pathway, as well as the role of chromatin remodeling in OCCC pathogenesis.

\section{ETHICS STATEMENT}

This study was carried out in accordance with the recommendations of the ethics committee in Region Hovedstaden (Denmark) and from the Lund University ethics committee (Sweden). All subjects gave written informed consent in accordance with the Declaration of Helsinki. The protocol was approved by the above agencies.

\section{AUTHOR CONTRIBUTIONS}

Study conception and design: NA, J-MJ, MN, and IH. Collection and assembly of data: NA, J-MJ, KB, AE, SW-F, AM, and SM. Data analysis and interpretation: NA, J-MJ, KB, AE, SW-F, AM, 
and IH. Manuscript writing: NA, J-MJ, and IH. Final approval of manuscript: all authors.

\section{ACKNOWLEDGMENTS}

The authors wish to thank Antti Ylipää, for critical review of the manuscript. This study was supported by grants from the Swedish Cancer Society, the GNilsson Cancer Foundation, Skåne University Hospital Research Foundation, the B Kamprad Foundation, and governmental funding of clinical research within the National Health Services (ALF). The funding agencies were not involved in design, data collection, analysis or interpretation, writing of the report, or the decision to submit the report for publication.

\section{REFERENCES}

1. Howlander N, Noone AM, Krapcho M, Garshell J, Miller D, Altekruse SF, et al. SEER Cancer Statistics Review, 1975-2011. (2014). Available from: http://seer. cancer.gov/csr/1975_2011/

2. Agarwal R, Kaye SB. Prognostic factors in ovarian cancer: how close are we to a complete picture? Ann Oncol (2005) 16(1):4-6. doi:10.1093/annonc/mdi104

3. Winter WE 3rd, Maxwell GL, Tian C, Carlson JW, Ozols RF, Rose PG, et al. Prognostic factors for stage III epithelial ovarian cancer: a Gynecologic Oncology Group Study. J Clin Oncol (2007) 25(24):3621-7. doi:10.1200/JCO.2006.10.2517

4. Winter WE 3rd, Maxwell GL, Tian C, Sundborg MJ, Rose GS, Rose PG, et al. Tumor residual after surgical cytoreduction in prediction of clinical outcome in stage IV epithelial ovarian cancer: a Gynecologic Oncology Group Study. J Clin Oncol (2008) 26(1):83-9. doi:10.1200/JCO.2007.13.1953

5. Prat J; FIGO Committee on Gynecologic Oncology. Staging classification for cancer of the ovary, fallopian tube, and peritoneum. Int J Gynaecol Obstet (2014) 124(1):1-5. doi:10.1016/j.ijgo.2013.10.001

6. Kurman RJ, Shih IeM. The origin and pathogenesis of epithelial ovarian cancer: a proposed unifying theory. Am J Surg Pathol (2010) 34(3):433-43. doi:10.1097/PAS.0b013e3181cf3d79

7. Lu Y, Cuellar-Partida G, Painter JN, Nyholt DR; Australian Ovarian Cancer Study, International Endogene Consortium (IEC), et al. Shared genetics underlying epidemiological association between endometriosis and ovarian cancer. Hum Mol Genet (2015) 24(20):5955-64. doi:10.1093/hmg/ddv306

8. Rauh-Hain JA, Winograd D, Growdon WB, Schorge JO, Goodman AK, Boruta DM, et al. Prognostic determinants in patients with uterine and ovarian clear carcinoma. Gynecol Oncol (2012) 125(2):376-80. doi:10.1016/j. ygyno.2011.12.221

9. Chan JK, Teoh D, Hu JM, Shin JY, Osann K, Kapp DS. Do clear cell ovarian carcinomas have poorer prognosis compared to other epithelial cell types? A study of 1411 clear cell ovarian cancers. Gynecol Oncol (2008) 109(3):370-6. doi:10.1016/j.ygyno.2008.02.006

10. Lee YY, Kim TJ, Kim MJ, Kim HJ, Song T, Kim MK, et al. Prognosis of ovarian clear cell carcinoma compared to other histological subtypes: a meta-analysis. Gynecol Oncol (2011) 122(3):541-7. doi:10.1016/j.ygyno.2011.05.009

11. Eltabbakh GH, Mount SL, Beatty B, Simmons-Arnold L, Cooper K. Clinical and molecular differences between clear cell and papillary serous ovarian carcinoma. J Surg Oncol (2006) 93(5):379-86. doi:10.1002/jso.20494

12. del Carmen MG, Birrer M, Schorge JO. Clear cell carcinoma of the ovary: a review of the literature. Gynecol Oncol (2012) 126(3):481-90. doi:10.1016/j. ygyno.2012.04.021

13. Yamashita Y, Nagasaka T, Naiki-Ito A, Sato S, Suzuki S, Toyokuni S, et al. Napsin A is a specific marker for ovarian clear cell adenocarcinoma. Mod Pathol (2015) 28(1):111-7. doi:10.1038/modpathol.2014.61

14. Kato N, Sasou S, Motoyama T. Expression of hepatocyte nuclear factor-1beta (HNF-1beta) in clear cell tumors and endometriosis of the ovary. Mod Pathol (2006) 19(1):83-9. doi:10.1038/modpathol.3800492

15. Yamaguchi K, Mandai M, Oura T, Matsumura N, Hamanishi J, Baba T, et al. Identification of an ovarian clear cell carcinoma gene signature that reflects inherent disease biology and the carcinogenic processes. Oncogene (2010) 29(12):1741-52. doi:10.1038/onc.2009.470

\section{FUNDING}

The Swedish Cancer Society (CAN2015/486), the G Nilsson Cancer Foundation (2016/728), Skåne University Hospital Research Foundation (1500291, 1600109), the B Kamprad Foundation (BKS 20/2015), and governmental funding of clinical research within the National Health Services (ALF) Region Skåne (2014/354).

\section{SUPPLEMENTARY MATERIAL}

The Supplementary Material for this article can be found online at http://journal.frontiersin.org/article/10.3389/fonc.2017.00109/ full\#supplementary-material.

16. Zorn KK, Bonome T, Gangi L, Chandramouli GV, Awtrey CS, Gardner GJ, et al. Gene expression profiles of serous, endometrioid, and clear cell subtypes of ovarian and endometrial cancer. Clin Cancer Res (2005) 11(18):6422-30. doi:10.1158/1078-0432.CCR-05-0508

17. Kanchi KL, Johnson KJ, Lu C, McLellan MD, Leiserson MD, Wendl MC, et al. Integrated analysis of germline and somatic variants in ovarian cancer. Nat Commun (2014) 5:3156. doi:10.1038/ncomms4156

18. Jones S, Wang TL, Shih IeM, Mao TL, Nakayama K, Roden R, et al. Frequent mutations of chromatin remodeling gene ARID1A in ovarian clear cell carcinoma. Science (2010) 330(6001):228-31. doi:10.1126/ science. 1196333

19. Chandler RL, Damrauer JS, Raab JR, Schisler JC, Wilkerson MD, Didion JP, et al. Coexistent ARID1A-PIK3CA mutations promote ovarian clear-cell tumorigenesis through pro-tumorigenic inflammatory cytokine signalling. Nat Commun (2015) 6:6118. doi:10.1038/ncomms7118

20. Gounaris I, Brenton JD. Molecular pathogenesis of ovarian clear cell carcinoma. Future Oncol (2015) 11(9):1389-405. doi:10.2217/fon.15.45

21. Friedlander ML, Russell K, Millis S, Gatalica Z, Bender R, Voss A. Molecular profiling of clear cell ovarian cancers: identifying potential treatment targets for clinical trials. Int J Gynecol Cancer (2016) 26(4):648-54. doi:10.1097/ IGC.0000000000000677

22. Elvin JA, Chura J, Gay LM, Markman M. Comprehensive genomic profiling (CGP) of ovarian clear cell carcinomas (OCCC) identifies clinically relevant genomic alterations (CRGA) and targeted therapy options. Gynecol Oncol Rep (2017) 20:62-6. doi:10.1016/j.gore.2017.02.007

23. Zannoni GF, Improta G, Chiarello G, Pettinato A, Petrillo M, Scollo P, et al. Mutational status of KRAS, NRAS, and BRAF in primary clear cell ovarian carcinoma. Virchows Arch (2014) 465(2):193-8. doi:10.1007/ s00428-014-1599-1

24. Schwartz DR, Kardia SL, Shedden KA, Kuick R, Michailidis G, Taylor JM, et al. Gene expression in ovarian cancer reflects both morphology and biological behavior, distinguishing clear cell from other poor-prognosis ovarian carcinomas. Cancer Res (2002) 62(16):4722-9.

25. Schaner ME, Ross DT, Ciaravino G, Sorlie T, Troyanskaya O, Diehn M, et al. Gene expression patterns in ovarian carcinomas. Mol Biol Cell (2003) 14(11):4376-86. doi:10.1091/mbc.E03-05-0279

26. Tsuchiya A, Sakamoto M, Yasuda J, Chuma M, Ohta T, Ohki M, et al. Expression profiling in ovarian clear cell carcinoma: identification of hepatocyte nuclear factor-1 beta as a molecular marker and a possible molecular target for therapy of ovarian clear cell carcinoma. Am J Pathol (2003) 163(6):2503-12. doi:10.1016/S0002-9440(10)63605-X

27. Sasano T, Mabuchi S, Kuroda H, Kawano M, Matsumoto Y, Takahashi R, et al. Preclinical efficacy for AKT targeting in clear cell carcinoma of the ovary. Mol Cancer Res (2015) 13(4):795-806. doi:10.1158/1541-7786.MCR-14-0314

28. Li M, Li H, Liu F, Bi R, Tu X, Chen L, et al. Characterization of ovarian clear cell carcinoma using target drug-based molecular biomarkers: implications for personalized cancer therapy. J Ovarian Res (2017) 10(1):9. doi:10.1186/ s13048-017-0304-9

29. JönssonJM,BartumaK,Dominguez-ValentinM,HarbstK,KetabiZ,MalanderS, et al. Distinct gene expression profiles in ovarian cancer linked to Lynch syndrome. Fam Cancer (2014) 13(4):537-45. doi:10.1007/s10689-014-9728-1 
30. Tavassoli FA, Devilee P. Pathology and Genetics of Tumours of the Breast and Female Genital Organs. Lyon: IARC Press (2003).

31. Silverberg SG. Histopathologic grading of ovarian carcinoma: a review and proposal. Int J Gynecol Pathol (2000) 19(1):7-15. doi:10.1097/00004347200001000-00003

32. Kurman RJ, Carcangiu ML, Herrington CS, Young RH. WHO Classification of Tumours of Female Reproductive Organs. Lyon: IARC Press (2014).

33. Heintz AP, Odicino F, Maisonneuve P, Quinn MA, Benedet JL, Creasman WT, et al. Carcinoma of the ovary. FIGO 26th Annual Report on the Results of Treatment in Gynecological Cancer. Int J Gynaecol Obstet (2006) 95(Suppl 1):S161-92. doi:10.1016/S0020-7292(06)60033-7

34. Edgar R, Domrachev M, Lash AE. Gene expression omnibus: NCBI gene expression and hybridization array data repository. Nucleic Acids Res (2002) 30(1):207-10. doi:10.1093/nar/30.1.207

35. Core Team R. R: A Language and Environment for Statistical Computing. Vienna: R Foundation for Statistical Computing (2014).

36. Saeed AI, Sharov V, White J, Li J, Liang W, Bhagabati N, et al. TM4: a free, opensource system for microarray data management and analysis. Biotechniques (2003) 34(2):374-8.

37. Tusher VG, Tibshirani R, Chu G. Significance analysis of microarrays applied to the ionizing radiation response. Proc Natl Acad Sci U S A (2001) 98(9):5116-21. doi:10.1073/pnas.091062498

38. Mi H, Muruganujan A, Thomas PD. PANTHER in 2013: modeling the evolution of gene function, and other gene attributes, in the context of phylogenetic trees. Nucleic Acids Res (2013) 41(Database issue):D377-86. doi:10.1093/nar/gks1118

39. Wang JZ, Du Z, Payattakool R, Yu PS, Chen CF. A new method to measure the semantic similarity of GO terms. Bioinformatics (2007) 23(10):1274-81. doi:10.1093/bioinformatics/btm087

40. Kamburov A, Pentchev K, Galicka H, Wierling C, Lehrach H, Herwig R. ConsensusPathDB: toward a more complete picture of cell biology. Nucleic Acids Res (2011) 39(Database issue):D712-7. doi:10.1093/nar/gkq1156

41. Afsari B, Fertig E. GSReg: Gene Set Regulation (GS-Reg), R Package Version 1.8.0. Baltimore, USA: The Sidney Kimmel Comprehensive Cancer Center, Johns Hopkins University School of Medicine (2014).

42. Jönsson JM, Johansson I, Dominguez-Valentin M, Kimbung S, Jönsson M, Bonde $\mathrm{JH}$, et al. Molecular subtyping of serous ovarian tumors reveals multiple connections to intrinsic breast cancer subtypes. PLoS One (2014) 9(9):e107643. doi:10.1371/journal.pone.0107643

43. Desmedt C, Haibe-Kains B, Wirapati P, Buyse M, Larsimont D, Bontempi G, et al. Biological processes associated with breast cancer clinical outcome depend on the molecular subtypes. Clin Cancer Res (2008) 14(16):5158-65. doi:10.1158/1078-0432.CCR-07-4756

44. McKenna A, Hanna M, Banks E, Sivachenko A, Cibulskis K, Kernytsky A, et al. The Genome Analysis Toolkit: a MapReduce framework for analyzing next-generation DNA sequencing data. Genome Res (2010) 20(9):1297-303. doi:10.1101/gr.107524.110

45. Cunningham F, Amode MR, Barrell D, Beal K, Billis K, Brent S, et al. Ensembl 2015. Nucleic Acids Res (2015) 43(Database issue):D662-9. doi:10.1093/nar/ gku1010

46. Paila U, Chapman BA, Kirchner R, Quinlan AR. GEMINI: integrative exploration of genetic variation and genome annotations. PLoS Comput Biol (2013) 9(7):e1003153. doi:10.1371/journal.pcbi.1003153

47. Komurov K, Ram PT. Patterns of human gene expression variance show strong associations with signaling network hierarchy. BMC Syst Biol (2010) 4:154. doi:10.1186/1752-0509-4-154

48. Hu P, Bader G, Wigle DA, Emili A. Computational prediction of cancer-gene function. Nat Rev Cancer (2007) 7(1):23-34. doi:10.1038/nrc2036

49. Fritz G, Just I, Kaina B. Rho GTPases are over-expressed in human tumors. Int J Cancer (1999) 81(5):682-7. doi:10.1002/(SICI)1097-0215(19990531)81:5< 682::AID-IJC2>3.0.CO;2-B

50. Canet B, Pons C, Espinosa I, Prat J. Ovarian clear cell carcinomas: RHO GTPases may contribute to explain their singular biologic behavior. Hum Pathol (2011) 42(6):833-9. doi:10.1016/j.humpath.2010.08.022
51. Tan TZ, Yang H, Ye J, Low J, Choolani M, Tan DS, et al. CSIOVDB: a microarray gene expression database of epithelial ovarian cancer subtype. Oncotarget (2015) 6(41):43843-52. doi:10.18632/oncotarget.5983

52. Takai M, Nakagawa T, Tanabe A, Terai Y, Ohmichi M, Asahi M. Crosstalk between PI3K and Ras pathways via protein phosphatase $2 \mathrm{~A}$ in human ovarian clear cell carcinoma. Cancer Biol Ther (2015) 16(2):325-35. doi:10.1080/153 84047.2014.1002362

53. Martin M, Geudens I, Bruyr J, Potente M, Bleuart A, Lebrun M, et al. PP2A regulatory subunit Balpha controls endothelial contractility and vessel lumen integrity via regulation of HDAC7. EMBO J (2013) 32(18):2491-503. doi:10.1038/emboj.2013.187

54. Chang CM, Chuang CM, Wang ML, Yang YP, Chuang JH, Yang MJ, et al. Gene set-based integrative analysis revealing two distinct functional regulation patterns in four common subtypes of epithelial ovarian cancer. Int J Mol Sci (2016) 17(8):E1272. doi:10.3390/ijms17081272

55. Tan DS, Iravani M, McCluggage WG, Lambros MB, Milanezi F, Mackay A, et al. Genomic analysis reveals the molecular heterogeneity of ovarian clear cell carcinomas. Clin Cancer Res (2011) 17(6):1521-34. doi:10.1158/10780432.CCR-10-1688

56. Tuefferd M, Couturier J, Penault-Llorca F, Vincent-Salomon A, Broët P Guastalla JP, et al. HER2 status in ovarian carcinomas: a multicenter GINECO study of 320 patients. PLoS One (2007) 2(11):e1138. doi:10.1371/journal. pone. 0001138

57. Lee CH, Huntsman DG, Cheang MC, Parker RL, Brown L, Hoskins P, et al. Assessment of Her-1, Her-2, And Her-3 expression and Her-2 amplification in advanced stage ovarian carcinoma. Int J Gynecol Pathol (2005) 24(2):147-52. doi:10.1097/01.pgp.0000152026.39268.57

58. Luoh SW, Ramsey B, Hanlon Newell A, Troxell M, Hu Z, Chin K, et al. HER-2 gene amplification in human breast cancer without concurrent HER-2 over-expression. Springerplus(2013) 2:386. doi:10.1186/2193-18012-386

59. McPherson LA, Loktev AV, Weigel RJ. Tumor suppressor activity of AP2alpha mediated through a direct interaction with p53. J Biol Chem (2002) 277(47):45028-33. doi:10.1074/jbc.M208924200

60. Anttila MA, Kellokoski JK, Moisio KI, Mitchell PJ, Saarikoski S, Syrjänen K, et al. Expression of transcription factor AP-2alpha predicts survival in epithelial ovarian cancer. Br J Cancer (2000) 82(12):1974-83. doi:10.1054/ bjoc. 2000.1146

61. Yamamoto S, Tsuda H, Takano M, Tamai S, Matsubara O. Loss of ARID1A protein expression occurs as an early event in ovarian clearcell carcinoma development and frequently coexists with PIK3CA mutations. Mod Pathol (2012) 25(4):615-24. doi:10.1038/modpathol. 2011.189

62. Li G, Ci W, Karmakar S, Chen K, Dhar R, Fan Z, et al. SPOP promotes tumorigenesis by acting as a key regulatory hub in kidney cancer. Cancer Cell (2014) 25(4):455-68. doi:10.1016/j.ccr.2014.02.007

63. Lee J, Kim DH, Lee S, Yang QH, Lee DK, Lee SK, et al. A tumor suppressive coactivator complex of p53 containing ASC-2 and histone H3-lysine-4 methyltransferase MLL3 or its paralogue MLL4. Proc Natl Acad Sci U S A (2009) 106(21):8513-8. doi:10.1073/pnas.0902873106

Conflict of Interest Statement: The authors declare that the research was conducted in the absence of any commercial or financial relationships that could be construed as a potential conflict of interest.

Copyright $\odot 2017$ Arildsen, Jönsson, Bartuma, Ebbesson, Westbom-Fremer, Måsbäck, Malander, Nilbert and Hedenfalk. This is an open-access article distributed under the terms of the Creative Commons Attribution License (CC BY). The use, distribution or reproduction in other forums is permitted, provided the original author(s) or licensor are credited and that the original publication in this journal is cited, in accordance with accepted academic practice. No use, distribution or reproduction is permitted which does not comply with these terms. 